\title{
Hf-W isotopic systematics of iron meteorites revisited
}

\author{
L. QIN', Q.ZHANG', N. DAUPHAS ${ }^{2}$, G. WANG ${ }^{3}, J^{\prime}$ LI $^{3}$
}

CAS Key Laboratory of Crust - Mantle Materials and Environments, and CAS Center for Excellence in Comparative Planetology, University of Science and Technology of China, Hefei, Anhui 230026, China (lpqin@ustc.edu.cn )

Origins Laboratory, Department of the Geophysical Sciences and Enrico Fermi Institute, The University of Chicago, Chicago, IL 60637, USA

Guangzhou Institute of Geochemistry, CAS, Guangzhou, Guangdong 510640, China

The ${ }^{182} \mathrm{Hf}-{ }^{182} \mathrm{~W}$ extinct radioactive chronomter has been proven to be extremely efficient for dating the relative timescales of metal-silicate differentiation processes in different planetary bodies in the early Solar System. However, the ${ }^{182} \mathrm{~W}$ abundances of iron meteorites can be shifted to negative values through capturing thermal neutrons under the radiation of galactic cosmic rays, resulting in apparent erronous chronological results. Various attempts have been made to correct for such effects on $\mathrm{W}$ isotopes, with most recent studies being focused on the use of the isotopic composition of platinum group elements (i.e. Pt and Os) as thermal neutron dose monitors. A potential problem with $\mathrm{Pt}$ isotopes is that potential nucleosynthetic anomaly on $\mathrm{Pt}$ has not been clearly ruled out, which may superimpose on the cosmogenic effect. While our former correction methods using ${ }^{10} \mathrm{Os} /{ }^{\circ} \mathrm{Os}$ or ${ }^{10} \mathrm{Os} /{ }^{1 \cdots} \mathrm{Os}$ ratios (internally normalized to $\left.{ }^{12} \mathrm{Os} /{ }^{18} \mathrm{Os}\right)$ as neutron dose monitor require the knowledge of the iron meteorites' chemical composition. In this work, we explore a new way of correcting for cosmogenic effects on $\mathrm{W}$ isotopes using Os isotopes by involving a new normalization scheme with ${ }^{12} \mathrm{Os}-{ }^{18} \mathrm{Os}$ as the internal normalization pair, and using the internally normalized ${ }^{\circ} \mathrm{Os} /{ }^{\circ} \mathrm{Os}$ ratio to monitor the effect of neutron-capture reactions on $\mathrm{W}$ isotopes. Consistent with modeling calculation results, we found that $\varepsilon^{12} \mathrm{~W}$ ${ }^{10} \mathrm{Os} /{ }^{\circ} \mathrm{Os}$ correlation slope is independent of the chemical composition of the iron meteorite of interest. Applying this new correction scheme to the W-Os isotopic data that we have obtained for a set of iron meteorites, we re-determined the pre-exposure ${ }^{182} \mathrm{~W}$ composition $\left(\varepsilon^{182} \mathrm{~W}_{0}\right)$ for different groups of irons. For IIAB $\left(\varepsilon^{122} \mathrm{~W}_{0}=-3.39 \pm 0.09\right)$, IIIAB $\left(\varepsilon^{182} \mathrm{~W}_{0}=-\right.$ $3.34 \pm 0.09)$ and IVB $\left(\varepsilon^{122} \mathrm{~W}_{0}=-3.13 \pm 0.07\right)$ groups, the values are consistent with previous studies using the W-Pt correction method. The IID $\left(\varepsilon^{182} \mathrm{~W}_{0}=-2.98 \pm 0.09\right)$ irons show a small discrepancy $(\sim 0.2 \varepsilon)$ with previous studies, which may reflect a potential $\mathrm{Pt}$ nucleosynthetic anomaly $\left(-0.15 \varepsilon\right.$ on $\left.{ }^{1 \mathrm{Pt}}\right)$. 